\title{
Editorial
}

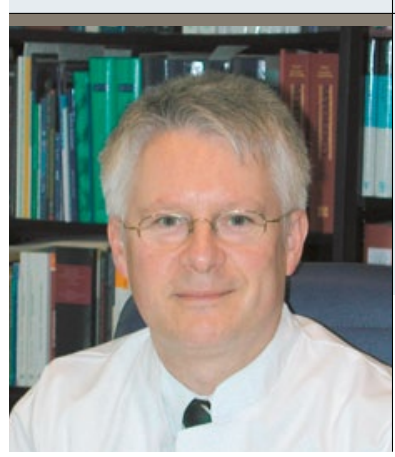

„Ob die Gabe von Statinen tatsächlich präventiv wirksam

ist, muss allerdings erst in größeren Studien randomisiert

und doppelblind untersucht werden."

\section{Die neue "Wunderwaffe" der Neurologie auf dem Prüfstand}

$\mathrm{D}$ ie vorliegende Ausgabe von DNP - Der Neurologe und Psychiater spannt erneut einen weiten, spannenden Bogen neuropsychiatrischer Krankheitsbilder.

Sehr häufig müssen Neurologen zusammen mit Kollegen aus der Hals-Nasen-Ohrenheilkunde sogenannten Schwindel behandeln. Hier ist es sehr wichtig, durch eine kluge und sorgfältige Anamneseerhebung zwischen systemischem und nicht systemischem Schwindel zu unterscheiden und sehr rasch die Weichen in Richtung HNO oder neurologisches Fachgebiet zu stellen. Die von Professor Strupp aus München verfasste Übersicht zur zertifizierten Fortbildung gibt einen hervorragenden Einblick über das, was „der Neurologe" heutzutage zu diesem sehr häufigen Symptom wissen sollte (siehe Seite 41). Schwindel kann im Rahmen peripherer Störungen wie beim Morbus Menière und Vestibularisausfall entstehen und äußert sich dann durch einen schweren Drehschwindel und Übelkeit. Zentrale Schwindelformen kommen bei der Migräne und häufig bei der Multiplen Sklerose vor. Die Diagnostik kann weitgehend durch geschicktes Untersuchen gestellt werden, nur selten liegen dem Schwindel schwere Erkrankungen zugrunde. Durch die Erfahrungen von Professor Strupp werden nicht nur die entsprechenden Symptome kritisch gewürdigt, sondern insbesondere auch eine sehr nützliche Übersicht über mögliche Therapien gegeben.

\section{Praxisrelevant: Leitlinien zur diabetischen Polyneuropathie}

Einen zweiten großen Block nimmt die Diskussion der diabetischen Polyneuropathie im Erwachsenenalter ein, die von Dr. von Stuckrad-Barre aus Wiesbaden verfasst wurde (siehe Seite 62 ). Die diabetische Polyneuropathie ist neben der alkoholischen Polyneuropathie die häufigste Ursache polyneuropathischer Beschwerden und verdient daher besondere
Berücksichtigung. Die Erkrankung kann sich in vielen Facetten äußern. Klassischerweise tritt sie distal symmetrisch im Sinne von Paraesthesien auf, kann aber auch Hirnnerven oder periphere Nerven im Sinne einer Mononeuropathie betreffen.

\section{Demenz auf dem Weg zur Volkskrankheit}

Weiterhin finden Sie in dieser Ausgabe zwei wichtige Beiträge zur Demenz, die vermutlich schon bald zur Volkskrankheit werden könnte und unser Gesundheitssystem auf eine schwere Probe stellen dürfte. Gerade wurde durch die Bundesregierung eine Verbesserung der Pflegesituation für Demenzkranke aufgelegt und die Regierung der Vereinigten Staaten von Amerika hat beschlossen, alles zu unternehmen, um die Ursachen und Therapie der Alzheimer-Demenz bis zum Jahre 2025 enträtselt zu haben.

In einem Beitrag zur zertifizierten Fortbildung von Dr. Froböse und Professor Kurz aus München, wird die frühe Diagnostik von Demenzerkrankungen propagiert, um rechtzeitig mit einer Therapie beginnen zu können, gut behandelbare Demenzen nicht zu übersehen und dem Patienten und seinem Umfeld die Möglichkeit zu geben, sich auf diese schwierige Situation einzustellen (siehe Seite 50).

\section{Statine als "Wunderwaffe"?}

In einem weiteren, sehr interessanten Artikel nehmen Dres. Luborzewski und Peters sowie Professor Heuser zu der neuen "Wunderwaffe" in der Neurologie, nämlich den Statinen, kritisch Stellung (ab Seite 59). Aus diesem Artikel ist zu entnehmen, dass eine frühzeitige Statinbehandlung (meist aufgrund einer Hypercholesterinämie) das Erkrankungsrisiko für die Alzheimer-Demenz statistisch senkt. Ob die Gabe von Statinen tatsächlich präventiv wirksam ist, müsste allerdings in größeren Studien randomisiert und doppelblind untersucht werden. Hoffnungsvoll stimmen diesbezüglich selbstverständlich die Beobach- 


\section{Scheibchenweise gegen die drohende Volkskrankheit "Demenz" - ob Statine in der Prävention von Nutzen sein können, muss sich aber erst in großen Studien erweisen.}

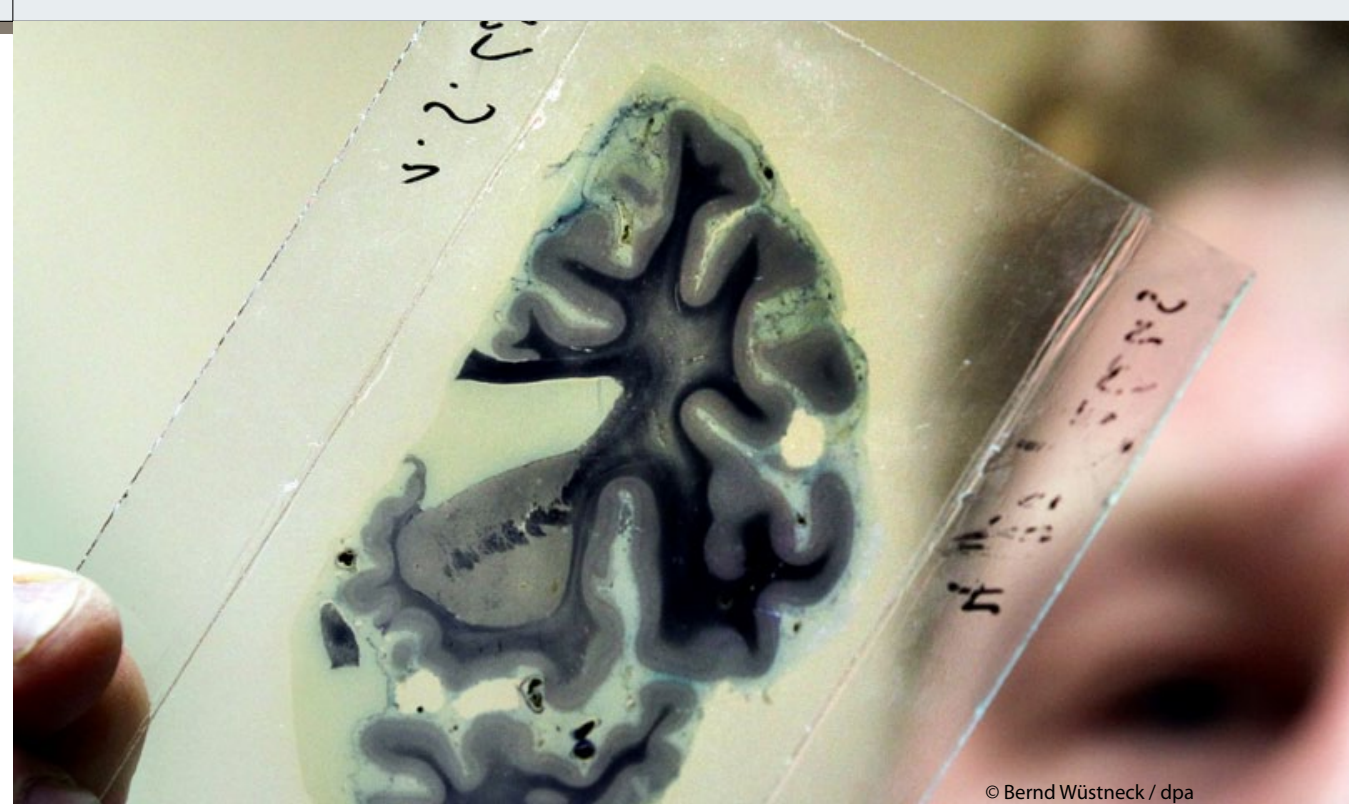

tungen, dass Statine zu einer Reduktion der zerebralen Amyloiddisposition führen und antiinflamma-torische Effekte aufweisen. Statine scheinen als Therapeutikum bei einer manifesten Alzheimer-Demenz nicht geeignet, viel wichtiger und interessanter ist aber, dass eine Statinbehandlung für die Prävention der Alzheimer-Demenz zumindest von Seiten der Wirkrationale genügend Basis für die oben geforderten Studien aufweist.

\section{Zwangsstörungen sind behandelbar}

Professor Voderholzer und Mitarbeiter aus Prien am Chiemsee haben die wichtigsten Studien des letzten Jahres zum Thema "Zwangsstörungen“ für Sie zusammengefasst und kommentiert. Insbesondere auf dem Gebiet der Psychotherapie- und der Versorgungsforschung sind interessante Neuerungen zu verzeichnen (ab Seite 22).

\section{Schizophrenie und Gewalt}

Im aktuellen Teil greift DNP - Der Neurologe und Psychiater das heikle Thema „Schizophrenie und Gewalt“ auf (siehe Seite 10). Durch den Fall des norwegischen Attentäters Anders Behring Breivik ist das Krankheitsbild der Schizophrenie auf brutale Weise in den Fokus der Öffentlichkeit geraten. Obgleich die Diagnose „paranoide Schizophrenie“ bei Breivik von vielen Fachleuten mittlerweile angezweifelt wird, stellt sich doch erneut die Frage: Inwieweit sind Gewalttaten bei einer Psychose das unmittelbare Resultat struktureller oder funktioneller Hirnveränderungen sind. Und: Sind die traditionellen psychopathologischen Modelle noch mit den fortschreitenden Erkenntnissen der Hirnforschung in Einklang zu bringen?Viel Spaß bei der Fortbildung wünscht Ihnen

Ihr

\section{Heinz Reichmann}

Professor Dr. med. Heinz Reichmann, FRCP, Klinikdirektor, Klinik und Poliklinik für Neurologie, Universitätsklinikum Carl Gustav Carus an der Technischen Universität Dresden

\section{Mehr zur Demenz auf springermedizin.de}

Nikotin hilft dem Gedächtnis auf die Sprünge

- In einer aktuellen Studie verbesserten sich mit Nikotinpflastern Kurzzeitgedächtnis und Aufmerksamkeit. *(2304256)

\footnotetext{
Alzheimer-Demenz durch stumme Hirninfarkte?

- Stumme Insulte gehen oft mit Gedächtnisproblemen und hirnorganischen Veränderungen einher, wie sie bei beginnendem Alzheimer typisch sind. Können sie gar eine Alzheimer-Demenz auslösen? * (2304784)
}

\author{
„Pathologischer" Liquor sagt Alzheimer voraus \\ - Schwedische Forscher haben herausgefunden, dass \\ sich mithilfe bestimmter Biomarker im Liquor rela- \\ tiv gut vorhersagen lässt, ob sich aus einer leichten \\ kognitiven Störung innerhalb der nächsten zehn Jahre \\ Alzheimer entwickeln wird. * (2291048)
* Diese Artikel finden Sie, indem Sie den Titel oder die (in Klammern gesetzte) ID-Nummer in \\ die Suche eingeben.
}

